\title{
India's Hidden Disease: Retrospective Study of Hansen Disease In A Tertiary Care Hospital
}

\author{
Evelyn Elizabeth Ebenezer*, Vimal Chander and S. Chitra
}

Department of Pathology in Saveetha Medical College, Chennai, Tamil Nadu, India

\begin{abstract}
Background: Leprosy is a chronic granulomatous disease caused by Mycobacterium leprae. There are various clinico-pathological forms of leprosy depending on the immune status of the host. Diagnosis of leprosy can be done by clinical, microbiological and histopathological examination. Histopathological examination is considered as important for confirmatory diagnosis, for assessment of the disease in patient's under treatment and also for research purposes

Methods: A total of 28 skin biopsies of leprosy patients were studied in the Department of Pathology in Saveetha medical college over 7 year's duration (2008 - 2015). A Ridley Jopling classification was used for the diagnosis and classification of the disease. All biopsies were stained with haematoxylin and eosin and Fite faraco. Clinico-histopathological correlation was done

Result: In this study, number of males (23cases) (82\%) were more than the number of females ( 5 cases) (18\%) Mean age of patients was 40 years. The Most common presentation of the lesion was hypopigmented macule involving two areas (13cases). Among 28 cases maximum cases were classified as borderline tuberculoid leprosy (8cases) (28.6\%) and least number of cases classified as histiod leprosy (1 case) $(3.6 \%)$. Fite faraco stain was positive for 3 cases in tuberculoid spectrum and 9 cases in lepromatous spectrum.

Conclusion: The current primary goal is early diagnosis of this disease in order to interrupt the transmission by early treatment. Histopathological examination is gold standard in confirmation of its diagnosis and its classification. As new immerging technique become available for the early diagnosis of leprosy, skin biopsies will continue to compliment these for providing more insights into the pathology of this disease.
\end{abstract}

Keywords: Leprosy, Tuberculoid Leprosy, Lepromatous Leprosy, Hansen, Ridley-Jopling Classification

\section{Introduction}

Leprosy is an infectious disease with history travelling as early as $600 \mathrm{BC}^{[1]}$ for cases of leprosy and has was considered as a curse to humankind. Leprosy is also known as Hansens disease named after Dr. Gerhard Henrik Armauer Hansen the person known for the numerous researches in Leprosy and proving it to be an infectious disease caused by a bacteria rather than a curse or sin to mankind. ${ }^{[2]}$ It is a chronic infectious disease caused by Mycobacterium leprae. It most commonly affects the superficial organs such as skin and peripheral nerves. Cases have been reported with presentation in other visceral organs such as liver, lymph node, bone marrow, eye, bones, and testes. ${ }^{[3]}$ The mode of transmission is through inhalation of bacilli that are excreted from the nasal passages of the multibacillary patient. There are incidences of direct person-to-person transmission through skin contact which may occur rare by transmission from hypodermic needles during skin tattooing or by physical trauma to the skin as has been reported. ${ }^{[3]}$ This disease presents with a long incubation period ranging from few weeks to 30 years with an average of 3 to 5 years. ${ }^{[1]}$
Depending on the immunity, clinical and histopathological features various types of leprosy may develop. ${ }^{[4]}$ Ridley and Jopling in 1966 proposed a histologic classification for leprosy based on the immunological spectrum into five groups that includes indeterminate leprosy (IL), Tuberculoid leprosy (TL), Borderline Tuberculoid leprosy (BT), Mid-Borderline leprosy (BB), Borderline lepromatous leprosy (BL) and Lepromatous leprosy (LL). ${ }^{[4]}$ It is further subdivided based on the number of acid fast bacilli present in the dermis that is expressed by the bacteriological index Graded from $1^{+}$to $6^{+}$depending on the number of bacilli seen ${ }^{[4]}$.

In spite of a steady fall reported in leprosy throughout the world, endemic countries such as India continue to be under the hidden burden of this disease. A total of 1,27,334 new cases were detected during the year 2015-16, which gives Annual New Case Detection Rate (ANCDR) of 9.71 per 100,000 population, as against $1,25,785$ cases in 2014-15. ${ }^{[5]}$ Showing an increase in incidence every year. It has been over a decade since leprosy was eliminated at the National level of India as a public health problem. ${ }^{\left[{ }^{6} \mathrm{H}\right.}$ However it is still considered a serious public health 
concern with social stigma. Hence this study has been conducted to enumerate the total number of leprosy cases diagnosed in skin biopsy specimens in Saveetha Medical College over a period of 8 years from 2008 to 2015 and to study the clinicohistopathological correlation.

\section{Materials and Methods}

This retrospective study was conducted in the Department of Pathology in Saveetha Medical College, Chennai, Tamil Nadu. All cases diagnosed after histopathology examination under the spectrum of leprosy in the department from the year 2008 to 2015 were included in the study. Cases where leprosy was suspected clinically but not confirmed on biopsy were excluded. Lepra reactions were excluded. The Case records, accompanying the biopsy specimens and issued reports were collected from the archives of the Department and data pertaining to age, sex, clinical information and histopathological findings were recorded. The Ridley-Jopling criteria was used to diagnose and classify leprosy clinically and histopathologically into the five groups as Tuberculoid leprosy (TL), Boderline tuberculoid(BT), Mid-borderline (BB), Borderline lepromatous (BL), Lepromatous leprosy(LL), Histoid leprosy(HL) and Inderminate (I).

Table 1: Spectrum of Clinical Diagnosis

\begin{tabular}{|l|c|c|}
\hline Clinical diagnosis & Cases & Percentage \\
\hline Indeterminate & 1 & $3 \%$ \\
\hline Tuberculoid & 4 & $14 \%$ \\
\hline Borderline tuberculoid & 12 & $43 \%$ \\
\hline Midborderline & 0 & $0 \%$ \\
\hline Borderline lepromatous & 3 & $11 \%$ \\
\hline Lepromatous leprosy & 4 & $14 \%$ \\
\hline Histoid leprosy & 1 & $4 \%$ \\
\hline Others & 3 & $11 \%$ \\
\hline Total & 28 & $100 \%$ \\
\hline
\end{tabular}

Table 2: Spectrum of Histopathological Diagnosis

\begin{tabular}{|l|c|c|}
\hline Histopthological diagnosis & Case No & Percentage \\
\hline Indeterminate & 3 & 11 \\
\hline Tuberculoid & 4 & $29 \%$ \\
\hline Borderline tuberculoid & 8 & $7 \%$ \\
\hline Mid borderline & 2 & $18 \%$ \\
\hline Borderline lepromatous & 5 & $18 \%$ \\
\hline Lepromatous leprosy & 5 & $3 \%$ \\
\hline Histoid leprosy & 1 & $100 \%$ \\
\hline Total & 28 & \\
\hline
\end{tabular}

All skin biopsies were fixed with $10 \%$ formalin. All the tissue were processed and embedded in paraffin blocks. Sections of $4 \mu \mathrm{m}$ thickness were cut and stained with hematoxylin and eosin and by modified Fite Farraco for Lepra bacilli. Slides were examined and reported using Ridley jopling classification and Bacillary index recorded.

\section{Result}

A total of 28 cases were studied over a duration of 7 years (2008-2015 May). There were 23 males and 5 females. Male: female ratio was 4.6:1. The age of the patients ranged from 11 to 81 years . Considering the incidence of cases reported, maximum cases were present in the year 2011 (6 cases) (Fig 1). The maximum Cases diagnosed in clinical and histopathology examination were borderline tuberculoid category both $42.9 \%$ and 28.6 respectively (table $1 \& 2$ ) (fig 2 ). The overall clinical and histopathological correlation was $53.6 \%$. Maximum correlation was seen in Borderline tuberculoid $87.5 \%$ and Borderline lepromatous 60\% (table 3). Fite Faraco stain demonstrated Acid Fast Bacilli in 13 cases (table 4). The AFB was mostly seen in Lepromatous spectrum and only three cases in tuberculoid spectrum (Fig 3). 
Table 3: Clinical and Histopathological Correlation

\begin{tabular}{|l|c|c|c|}
\hline HPE cases & Correlating & Noncorrelating & Correlation \% \\
\hline Indeterminate & 1 & 2 & 33.3 \\
\hline Tuberculoid & 2 & 2 & 50 \\
\hline Borderline tuberculoid & 7 & 1 & $87.5 \%$ \\
\hline Borderline borderline & 0 & 2 & 0 \\
\hline Borderline lepromatous & 3 & 2 & 60 \\
\hline Lepromatous leprosy & 2 & 3 & 40 \\
\hline Histoid leprosy & 0 & 1 & 0 \\
\hline Overall correlation & 15 & 13 & $53.6 \%$ \\
\hline
\end{tabular}

Table 4: Fite Farraco Correlation

\begin{tabular}{|c|c|c|}
\hline Fite Faraco & Tubercuoid Spectrum & Lepromatous Spectrum \\
\hline Present & 3 & 10 \\
\hline Absent & 9 & 1 \\
\hline Total & 12 & 11 \\
\hline
\end{tabular}

Table 5: Comparative analysis of correlation in different histopathology types done by different authors.

\begin{tabular}{|c|c|c|c|c|c|c|c|c|}
\hline $\begin{array}{l}\text { Type of } \\
\text { leprosy }\end{array}$ & 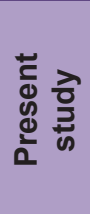 & $\begin{array}{l}\frac{\Xi}{\sqrt{0}} \\
\frac{ \pm}{0} \\
\frac{2}{\pi} \\
\frac{10}{3}\end{array}$ & 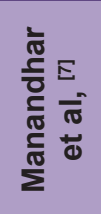 & $\begin{array}{l}\text { Mathur et } \\
\quad \text { al }{ }^{[9]}\end{array}$ & 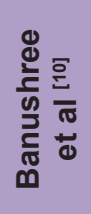 & 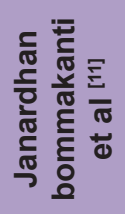 & 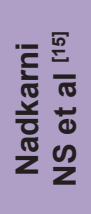 & 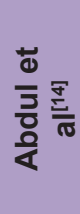 \\
\hline TT & 50 & 66 & 24 & 73.2 & 81 & 33.3 & 97 & 100 \\
\hline BT & 87.5 & 68.1 & 63.1 & 89.7 & 83.3 & 65.9 & 95 & 77.8 \\
\hline BB & 0 & - & 0 & 64.7 & - & 0 & 89 & - \\
\hline $\mathrm{BL}$ & 60 & 87.5 & 57.14 & 72.4 & 90 & 20.2 & 87 & 0 \\
\hline LL & 40 & 50 & 57.14 & 95.2 & 100 & 60 & 98 & 42.9 \\
\hline $\begin{array}{l}\text { Overall } \\
\text { concordance }\end{array}$ & 53.6 & 54 & 45.3 & 80.4 & 79.4 & 56 & 81.8 & 62.6 \\
\hline
\end{tabular}

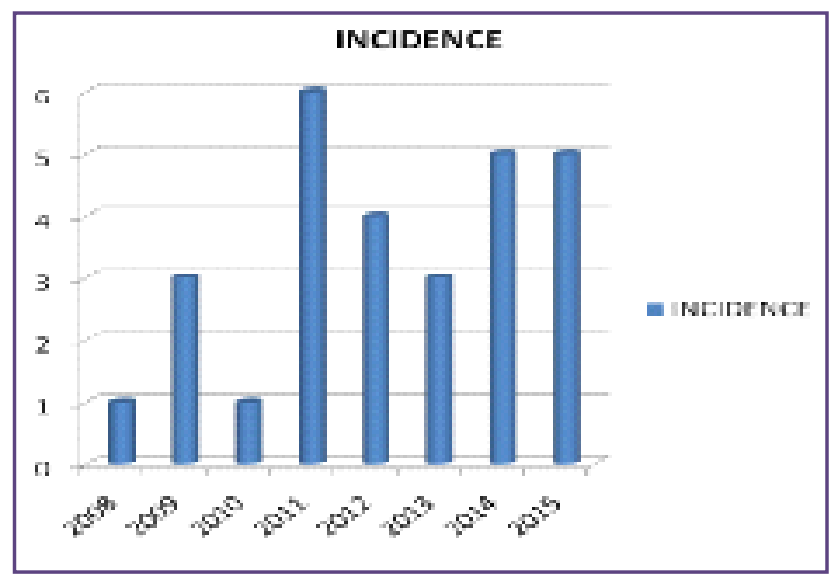

Fig 1: Incidence of cases of leprosy

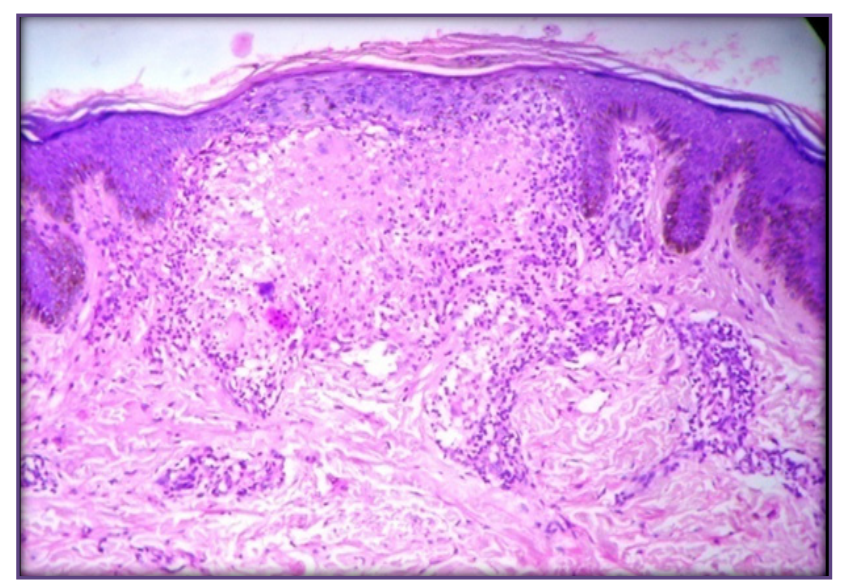

Fig 2: 10X, H and E: subepidermal well defined granuloma

Annals of Pathology and Laboratory Medicine, Vol. 5, Issue 1, January, 2018 


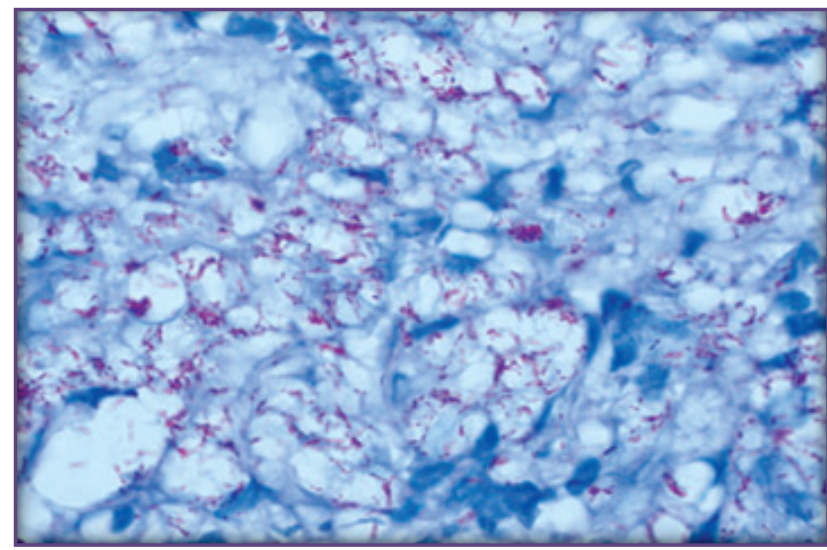

Fig 3: Fite Faraco: Acid Fast Bacilli seen in LL ( 100x10x)

\section{Discussion}

Leprosy is an example of a single disease that represents a spectrum of forms related to the cell-mediated immune response to mycobacterium leprae, which determines the classification and prognosis of the disease. The most commonly accepted classification is that of Ridley and Jopling, Based on immunity but has been correlated with clinical, histopathological and bacteriological finding. Even after such elaborate classification disparity prevails between the clinical diagnosis and histopathological features.

In our study, 28 cases were diagnosed with leprosy of which male were $82 \%$ and women $17 \%$.Male: Female ratio was 4.6:1 . Different studies have proved that leprosy was more common in males than in females. The age of the patients ranged from 11 to 81 years with most common presentation in the age group of 30-40years.Decreased cases in children may indicate a decrease in the incidence of leprosy. ${ }^{[4]}$

There was a complete agreement with clinical and histopathological diagnosis with 15 cases. The overall clinicopathological concordance was $53.6 \%$ similar to the studies done by Tiwari et al 54\%, ${ }^{[4]}$ Janardhan Bommakanti et al $56 \%,{ }^{[11]}$ Sharma et al $53.44 \%,{ }^{[12]}$ Pandya et al $58.13 \%,{ }^{[13]}$ and Mithra et al 53\% ${ }^{[16]}$ Various studies showed clinicopathological concordance from $45.3 \%$ to $80.4 \%$.

BT cases were the most common. The borderline groups (tuberculoid and lepromatous) constituted more than $50 \%$ of the cases. The most common clinical and histopathological presentation was borderline tuberculoid with $87.5 \%$ concordance ( 8 cases). Many other studies have also shown common presentation of borderline tuberculoid spectrum. Histopathological diagnosis of borderline tuberculoid was based on the presence of Clear subepidermal zone with epithelioid granuloma surrounded by peripheral lymphocytes and few Langhans giant cells. 3 cases were positive for AFB with a bacillary index of $+1 /+2$. Similar concordance was seen in other studies done by Mathur et al $89.74 \%,{ }^{[9]}$ Banushree et al $83.3 \%{ }^{[10]}$ (Table 5)

In case of Borderline lepromatous leprosy, the overall correlation was $60 \%$ (3 cases). The histopathological diagnosis of borderline lepromatous was based on Loose ill-defined granulomas composed of histiocytic cells with dense lymphocytic infiltrates. AFB was positive in 3 cases with an Bacillary index of $2+$ to $5+$. Similar concordance was seen in a study done by Manandhar et al $57.14 \%$. Other studies showed concordance ranging from $57 \%$ to $90 \% .4$ cases were histopathologically diagnosed as Tuberculoid leprosy with a clinicopathological correlation of $50 \%$. The diagnosis of tuberculoid leprosy was given on the basis of well defined epithelioid granuloma with Langhans giant cells surrounded by dense lymphocytic infiltrates. AFB was negative in all these cases. Some studies showed a clinicopathological correlation ranging from $24 \%$ to $100 \%$. This variable presentation may be due to the immune status, morphological changes and biopsy techniques.

In case of lepromatous leprosy, the clinicopathological correlation was $40 \%$ ( 1 case). One case clinically diagnosed as lupus vulgaris was reported on histopathology as lepromatous leprosy. The histopathologic diagnosis of lepromatous leprosy was based on the presence of Grenz zone with diffuse arrangement of histiocytes and foamy macrophages replacing skin adnexal structures. AFB was positive in all the cases with bacillary index ranging from $3+$ to $5+$. Similar concordance was also seen in study done by Abdul et al $42.9 \% \cdot{ }^{[14]}$ Other studies have shown higher concordance in the range of $50-100 \%$.

Histoid leprosy was clinically diagnosed as lepromatous leprosy and reported as histoid on histopathologic examination with a Bacillary index of $5+$.

In case of Indeterminate leprosy, only one case was clinically diagnosed as indeterminate and the other two cases were not further classified in the Ridley-Jopling classification. Bacillary index ranged from $1+$ to $2+$. Indeterminate leprosy cannot be classified within Ridley-Jopling spectrum due to lack of distinguishing features like absence of granuloma.

The least clinicopathologic correlation was noted in midborderline leprosy and similar results were obtained was also noted by Manandhar et al, ${ }^{[7]}$ Sharma et al, ${ }^{[1]}$ Moorthy et al. ${ }^{[16]}$. This is due to the highly unstable immune status. In contrast to our result, Nayak SV et al showed maximum correlation in midboderline cases with a concordance of $100 \% \cdot{ }^{[15]}$

This variation of results observed with other studies may be due to different criteria used to select the cases and difference 
in the number of cases of each type. The other factors that also play a significant role are Clinical information such as site of lesion, type of lesion, nerve involvement, sensory impairment, treatment history along with immunological status of patients as it is very important for the pathologist to correlate histopathologically. Histopathological diagnosis may also vary depending on various factors such as Duration of the lesion, size of the biopsy specimen, depth of biopsy taken, quality of section. The most important difference is due to interobserver variations which has a major role in the clinico-pathological evaluation. The disparity between clinical and histological correlation was also observed to be due to the well-defined histopathology features which were precise and also consider the immunological response of the tissue while clinical diagnosis is only based on the clinical presentation of the lesion. Moreover, a group of leprosy cases $(\mathrm{BT}+\mathrm{BB}+\mathrm{BL})$ have continual modification in their immunologic response and histologic classification gives a better indication for the typing and subclassification.

\section{Conclusion}

The current primary goal is early diagnosis of this disease in order to interrupt the transmission by early treatment. Histopathological examination is gold standard in confirmation of the diagnosis and its classification. Correlation of histopathology features with bacteriological index and clinical findings is necessary for accurate classification of leprosy. As new emerging techniques become available for the early diagnosis of leprosy, skin biopsies will continue to compliment these for providing more insights into the pathology of this disease. It also gives indication of progression or regression of the disease under treatment.

\section{Reference}

1. Nadia, S., Rashmi, J., Sohaib, A., Rawat, S.D.S., Thamarai, S.N., and Meena, H. Clinico pathological correlation of leprosy: a 4 years retrospective study from a tertiary referral centre in North India. International Journal of Medical Research \& Health Sciences 2015;4:350-354.

2. Jay, V. The legacy of Armauer Hansen. Archives of Pathology and Laboratory Medicine 2000;124(4):496-497.

3. Singh A, Xiaoman Weng and Indira Nath (2011). Skin Biopsy in Leprosy, Skin Biopsy - Perspectives, Dr. Uday Khopkar (Ed.), ISBN: 978-953-307-290-6, InTech, Available from: http://www.intechopen.com/books/skinbiopsy-perspectives/skin-biopsy-in-leprosy
4. Tiwari M, Ranabhat S, Maharjan S. Clinicohistopathological correlation of leprosy: A retrospective study of skin biopsy specimens in Chitwan Medical College. Inter J Medical Sci Res Prac 2015;2(1):8-11.

5. National Leprosy Eradication Program (NLEP). NLEP Annual report for the year 2015-16. Nirman Bhavan, New Delhi: Central Leprosy Division, Directorate General of Health Services, Ministry of Health and Family Welfare (GoI); 2016. Available from: http://nlep.nic.in/pdf/revised $\% 20$ annual\%20report\%2031st\%20March\%202015-16.pdf

6. Shoba KL, Prakash CJ. Clinico-Histopathological Study of Leprosy. International Journal of Scientific Study 2015;3(1):94-98.

7. Manandhar U, Adhikari RC, Sayami G. Clinicohistopathological correlation of skin biopsies in leprosy. Journal of Pathology of Nepal 2013;3:452-458.

8. Bijjaragi S, Kulkarni V, Suresh KK, Chatura KR and Kumar P. Correlation of clinical and histopathological classification of Leprosy in post elimination era. Indian $\mathbf{J}$ Lepr 2012;84:271-275.

9. Mathur MC, Ghimire RBK, Shrestha P, Kedia SK. Clinicohistopathological Correlation in Leprosy Kathmandu Univ Med J 2011;36(4):248-51.

10. BanushreeCS,BhatRV,UdayashankarC.Clinicopathological correlation of Hansen's disease: a retrospective study of skin Biopsies. Indian Journal of Pathology and Oncology 2016;3(3);491-495.

11. Bommakanti J, Putta S, Gokhale S. Histopathological Relevance in Clinical Spectrum of Hansen's Disease. Journal of medical science and clinical research 2016;4(12): 14678-84.

12. Sharma A, Sharma RK, KC Goswami KC, Baradwaj S. Clinicohistological correlation of leprosy. JK Science 2008;10:120-4.

13. Pandya AN, Tailor HJ. Clinicohistopathological correlation of leprosy. Indian J Dermatol Venereol Leprol 2008;74(2):174-6.

14. Lakshmy R, Samad KA, Nandakumar G, et al. A study of clinico-pathological concordance in patients with Hansen's disease across the spectrum. Journal of Evolution of Medical and Dental Sciences 2017;6(49):3742-5.

15. Nadkarni NS, Rege VL. Significance of histopathological classification in leprosy. Ind J Lepr 1999;71:325-32.

16. Mitra K, Biswas S, Saha B, Dasgupta A. Correlation between clinical and Histopathological criteria for the classification of Leprosy. Ind J Dermatol 46(3):135-7.

*Corresponding author:

Evelyn Elizabeth Ebenezer, No 13 venkatesan street east tambaram Chennai-(India) - 59

Phone: +91 9677245150

Email: evelyneby@gmail.com

Financial or other Competing Interests: None.

Date of Submission : 08.09.2017

Date of Acceptance : 10.10.2017

Date of Publication : 28.01.2018 